\title{
BIOENSAIOS PARA IDENTIFICAÇÃo DE BIÓTIPOS DE Euphorbia heterophylla COM RESISTÊNCIA MÚLTIPLA A INIBIDORES DA ALS E DA PRotox ${ }^{1}$
}

\author{
Greenhouse and Laboratory Bioassays for Identification of Euphorbia heterophylla Biotypes \\ with Multiple Resistance to Protox and ALS-Inhibiting Herbicides
}

\author{
TREZZI, M.M. ${ }^{2}$, VIDAL, R.A. ${ }^{3}$, KRUSE, N.D. ${ }^{4}$ e NUNES, A.L. ${ }^{5}$
}

\begin{abstract}
RESUMO - Quatro bioensaios, dois em casa de vegetação e dois em laboratório, foram conduzidos com o objetivo de identificar biótipos de Euphorbia heterophylla (EPHHL) com resistência múltipla a inibidores da ALS e da PROTOX. Em casa de vegetação, plantas do biótipo suscetível (S) e dos biótipos 4 e 23, suspeitos de resistência múltipla, foram aspergidas com diferentes doses de imazethapyr e fomesafen. Nos bioensaios em laboratório, sementes dos biótipos S e 4 foram depositadas em placas de Petri contendo diferentes concentrações dos mesmos herbicidas. Curvas de dose-resposta foram ajustadas, utilizando os modelos logístico e polinomial, respectivamente, para os dados obtidos em casa de vegetação e em laboratório. Em casa de vegetação, o fator de resistência (FR) a imazethapyr para o biótipo 4 foi superior a 24, enquanto para o biótipo 23 ele foi de 15. Os FRs a fomesafen foram, respectivamente, de 62 e 39, para os mesmos biótipos. Em um período de 144 horas, concentrações de imazethapyr e fomesafen no bioensaio em laboratório foram capazes de discriminar os crescimentos da parte aérea e radicular dos biótipos de EPHHL com resistência múltipla e S. Os resultados confirmam ser tanto os testes em casa de vegetação quanto os laboratoriais, utilizando placas de Petri, metodologias apropriadas para discriminar biótipos de EPHHL S daqueles com resistência múltipla.
\end{abstract}

Palavras-chave: metodologia, planta daninha, resistência a herbicidas.

ABSTRACT - Four bioassays were developed under greenhouse and laboratory conditions to identify Euphorbia heterophylla (EPHHL) biotypes with multiple resistance to PROTOX and ALS-inhibiting herbicides. In the greenhouse bioassays, plants of a susceptible (S) biotype and of two biotypes suspected of multiple resistance (\#4 and \#23) were sprayed using different levels of the herbicides imazethapyr and fomesafen. The laboratory bioassays consisted of a comparative test between biotypes $S$ and \#4, exposed to germination in imazethapyr and fomesafen solutions. Dose-response relationships were adjusted using logistic and polynomial models, respectively, for data obtained from greenhouse and laboratory bioassays. For biotype \#4, the resistance factor (RF) to imazethapyr was above 24, while for biotype \#23 it was 15. FR to fomesafen were, respectively, 62 and 39, for the same biotypes. In the laboratory bioassay, a period of 144 hours was sufficient to discriminate differencial growth response to imazethapyr and fomesafen levels between $R$ and $S$ biotypes. The results confirm that both greenhouse and laboratory tests with Petri dishes are appropriate methodologies to discriminate $S$ and multiple $R$ EPHHL biotypes.

Keywords: methodology, weed, resistance to herbicides.

\footnotetext{
Recebido para publicação em 10.3.2006 e na forma revisada em 4.8.2006.

2 Eng.-Agrônomo, Dr., Curso de Agronomia, Universidade Tecnológica Federal do Paraná - UTFPR, Campus Pato Branco,Via do Conhecimento, km 01, Caixa postal 571, 85503-390 Pato Branco-PR. ${ }^{3}$ Eng.-Agrônomo, Ph.D., Faculdade de Agronomia, Universidade Federal do Rio Grande do Sul-UFRGS, Av. Bento Gonçalves, 7712, Caixa Postal 15100, 91540-000 Porto AlegreRS. ${ }^{4}$ Eng.-Agrônomo, Dr., Centro de Ciências Rurais, Universidade Federal de Santa Maria - UFSM, 97105-900 Santa MariaRS. ${ }^{5}$ Eng.-Agrônomo, mestrando, Programa de Pós-Graduação em Fitotecnia, UFRGS.
} 


\section{INTRODUÇÃO}

Em geral, biótipos de plantas daninhas resistentes (R) a herbicidas podem ser controlados com o uso de herbicidas com mecanismo de ação alternativo aos geradores da resistência. No entanto, a pressão de seleção gerada pelo uso do mecanismo alternativo pode levar ao surgimento de biótipos com resistência a mais de um mecanismo de ação.

No mundo e também no Brasil, o mecanismo de ação com maior número de casos de biótipos R é o dos inibidores da enzima acetolactato sintase (ALS). No Brasil, atualmente, biótipos resistentes a inibidores da ALS são documentados em Bidens pilosa, Bidens subalternans, Euphorbia heterophylla (EPHHL), Cyperus difformis, Fimbristylis miliacea, Parthenium hysterophorus, Raphanus sativus e Sagittaria montevidensis (Christofolleti et al., 1996; Gazziero et al., 1998; Noldin et al., 1999; Vidal \& Merotto Jr., 1999; Monqueiro et al., 2000; Noldin et al., 2002a, b). No mundo, a inibição da enzima ALS também é o mecanismo de ação envolvido no maior número de casos de resistência a mais de um mecanismo de ação (aproximadamente 20 espécies e 35 biótipos) (Heap, 2006).

Recentemente, em 2004, em áreas cultivadas com soja localizadas na região sudoeste do Paraná, foram identificados dois biótipos de EPHHL que não respondiam à aplicação das doses recomendadas dos herbicidas imazethapyr (inibidor da ALS) e fomesafen (inibidor da PROTOX) para o controle dessa espécie (Trezzi et al., 2005). Essas áreas apresentavam histórico de aplicação por vários anos de herbicidas inibidores da ALS e da PROTOX.

Casos de resistência de EPHHL a mais de um mecanismo de ação geram bastante apreensão no meio agrícola, pois os principais herbicidas seletivos para a cultura da soja, capazes de controlar plantas resistentes a inibidores da ALS em pós-emergência, são compostos que inibem a enzima PROTOX (Merotto Jr. \& Vidal, 2001). Se a resistência de EPHHL a um mecanismo de ação representa uma grande ameaça à produção de culturas em áreas infestadas, a resistência simultânea a inibidores da ALS e da PROTOX representa ameaça muito maior, pois limita ainda mais as opções de controle, com conseqüente necessidade de utilização de outras práticas de manejo e potencial para reduzir a produtividade das culturas. Na busca de sistemas mais eficazes de controle, sojicultores com problemas de EPHHL resistente a inibidores da ALS e/ou com resistência simultânea a inibidores da ALS e da PROTOX vêm utilizando soja com o gene RR em suas lavouras, bem como intensificando o uso de sistemas de rotação com outras culturas.

Biótipos de leiteira (E. heterophylla) com resistência a inibidores da ALS foram identificados em vários estados do Brasil e no Paraguai (Heap, 2006). No mundo, os casos de resistência a herbicidas inibidores da ALS e PROTOX, simultaneamente, estão restritos, até o momento, a biótipos de Amaranthus spp., identificados nos estados do Kansas (Shoup et al., 2003) e Illinois, nos Estados Unidos. Até o momento, as hipóteses de que a absorção, translocação e metabolização diferencial sejam os mecanismos de resistência a inibidores da PROTOX para esses biótipos de Amaranthus spp. estão descartadas (Li et al., 2004), sendo uma modificação no local de ação a provável causa da resistência (Tranel et al., 2006).

Várias abordagens podem ser utilizadas para identificação de biótipos R, como bioensaios em placas de Petri, ensaios com as partes aéreas, ensaios com a enzima-alvo etc. (Beckie et al., 2000). Os ensaios mais amplamente utilizados são os de dose-resposta, normalmente realizados em condições de casa de vegetação. Esses ensaios utilizam doses subletais e letais de herbicidas para, através de equações não-lineares, estimar a $I_{50}$ (dose necessária para reduzir o desenvolvimento em 50\%) do biótipo suscetivel e do biótipo potencialmente resistente (Seefeldt et al., 1995). Trabalhos em laboratório também podem auxiliar na determinação de curvas de dose-resposta, estratégia importante para a definição mais rápida de diferenças entre biótipo suscetível e resistente (Carvalho et al., 2004).

Os objetivos deste trabalho foram confirmar a resistência múltipla a ALS e PROTOX em biótipos de $E$. heterophylla e verificar se a técnica do bioensaio em placas de Petri é eficiente para discriminar a resposta entre biótipos dessa espécie suscetíveis e com resistência múltipla. 


\section{MATERIAL E MÉTODOS}

Para desenvolver os ensaios em casa de vegetação, em 2003 foram coletadas amostras de sementes de EPHHL em duas áreas com suspeita de resistência múltipla a inibidores da ALS e PROTOX, pertencentes aos municípios de Pato Branco (biótipo 23) e Vitorino (biótipo 4), na região sudoeste do Paraná. Em 2004, na área pertencente ao município de Vitorino (biótipo 4), procedeu-se à amostragem georreferenciada de solo em área com infestação de EPHHL em 20 pontos distintos, com a finalidade de identificar com maior precisão os locais em que efetivamente havia o problema. Em casa de vegetação, o solo coletado e contaminado com sementes de EPHHL foi colocado sobre telhas de zinco e irrigado periodicamente, para promover a emergência das plantas. Quando as plantas de EPHHL estavam com duas folhas verdadeiras, foram aspergidas com imazethapyr (100 g i.a. ha-1) e fomesafen (250 g i.a. ha-1). Em 2005, foram identificados os pontos georreferenciados, correspondentes à existência de plantas sobreviventes no teste anterior, procedendo-se novamente à coleta de sementes com resistência múltipla na área de lavoura, sendo estas utilizadas nos ensaios em placas de Petri, no laboratório.

Nos dois ensaios conduzidos, foi utilizado o delineamento completamente casualizado, arranjado em um fatorial hierárquico, com três repetições. No primeiro ensaio, o fator A representou os biótipos de EPHHL (4 e S); o segundo fator, os herbicidas (imazethapyr e fomesafen); e o terceiro, as doses dos herbicidas. No segundo ensaio, os biótipos comparados foram o 23 e o S, sendo os demais fatores os mesmos do primeiro ensaio.

Aproximadamente 50 sementes de EPHHL dos biótipos 4 e 23 foram semeadas em vasos contendo $500 \mathrm{~cm}^{3}$ de solo argiloso. Sementes de uma população $\mathrm{S}$, provenientes de plantas originárias de Pato Branco, foram utilizadas como testemunha. As plântulas foram mantidas sob irrigação e, quando estavam com uma folha verdadeira, procedeu-se ao desbaste para 15 plantas por vaso. Quatro dias após, quando estas plantas estavam com duas folhas, foram aplicados os herbicidas imazethapyr e fomesafen.
No primeiro ensaio, plantas do biótipo $\mathrm{S}$ foram aspergidas com $0,2,6,6,4,16,40 \mathrm{e}$ $100 \mathrm{~g} \mathrm{ha}^{-1}$ de imazethapyr ou $0,6,4,16,25$, 100 e $250 \mathrm{~g} \mathrm{ha}^{-1}$ de fomesafen, enquanto o biótipo 4 foi aspergido com 0, 100, 200, 400 e $800 \mathrm{~g} \mathrm{ha}^{-1}$ de imazethapyr e 0, 250, 500, 1.000 e $2.000 \mathrm{~g} \mathrm{ha}^{-1}$ de fomesafen. No segundo ensaio, plantas do biótipo $\mathrm{S}$ foram aspergidas com os mesmos níveis utilizados no primeiro ensaio, enquanto as do biótipo 23 foram aspergidas com 0, 120, 240, 480, 960 e $1.920 \mathrm{~g} \mathrm{ha}^{-1} \mathrm{de}$ imazethapyr e $0,300,600,1.200,2.400$ e $4.800 \mathrm{~g} \mathrm{ha}^{-1}$ de fomesafen.

Quarenta dias após a aplicação dos tratamentos (DAT), as partes aéreas das plantas foram coletadas e secas a $60^{\circ} \mathrm{C}$, durante $48 \mathrm{~h}$, para determinação da matéria seca (MS).

Os dados foram submetidos à análise de variância, pelo teste $\mathrm{F}$. A relação da MS da parte aérea com os níveis de herbicidas foi ajustada por regressão, empregando-se inicialmente o modelo logístico de quatro parâmetros, utilizando-se o programa computacional Sigmaplot:

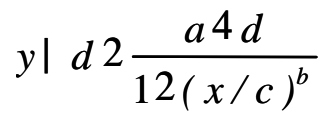

O procedimento adotado pelo programa estima, por sucessivas iterações, os valores dos parâmetros nos quais a soma dos quadrados dos desvios das observações, em relação aos valores ajustados, é mínima. Nesse modelo, $y$ representa a variável dependente, $x$ a concentração do herbicida e $a, b, c$ e $d$ são coeficientes da curva, em que $a$ é a média da resposta da testemunha, $b$ representa a declividade da curva, c é a concentração que proporciona 50\% do valor da variável dependente $\left(\mathrm{I}_{50}\right)$ e $d$ é a média da resposta sob doses elevadas (Seefeldt et al., 1995). Em caso de falta de ajustamento ao modelo de quatro parâmetros, foi testado o ajustamento ao modelo logístico de três parâmetros, o qual não inclui o parâmetro $d$ :

$$
y \frac{a}{1 \square(x / c)^{b}}
$$

Os fatores de resistência (FRs) foram calculados por meio do quociente entre os parâmetros $c\left(\mathrm{I}_{50}\right)$ das equações logísticas dos biótipos R e S. 
Quanto aos dois ensaios conduzidos em laboratório, neles se utilizou o delineamento completamente casualizado, com três repetições, arranjado em fatorial $2 \times 9$. Os testes foram conduzidos em câmara de crescimento tipo BOD, sob temperatura diurna/noturna de $29 / 25{ }^{\circ} \mathrm{C}$ e fotoperíodo de $12 \mathrm{~h}$. Vinte sementes de EPHHL do biótipo 4, coletadas em área previamente selecionada e testada quanto à resistência múltipla, foram semeadas sobre papel-filtro embebido em $5 \mathrm{~mL}$ de água ou sob solução com herbicida, em placas de Petri com $9 \mathrm{~cm}$ de diâmetro. Sementes de uma população $\mathrm{S}$, originária de Pato Branco, foram utilizadas como testemunha.

No primeiro ensaio, avaliou-se a resposta dos biótipos de EPHHL S e 4 às concentrações de $0,2,5,5,10,20,40,80,160$ e $320 \mathrm{mg}$ de imazethapyr por litro. No segundo ensaio, foi avaliada a resposta dos mesmos biótipos às concentrações de $0,625,1.250,1.875$, $2.500,3.125,3.750,4.375$ e $5.000 \mathrm{mg}$ de fomesafen por litro. As concentrações dos herbicidas utilizados nos ensaios foram selecionadas através da condução de experimentos preliminares.

Após 144 horas de embebição, foram determinados os percentuais finais de germinação das sementes e os comprimentos das partes aéreas (COMPA) e das radículas (COMRA) das plântulas emergidas. Foram consideradas emergidas apenas as plântulas cujas radículas ultrapassaram o comprimento de $2 \mathrm{~mm}$ (Voll et al., 2003).

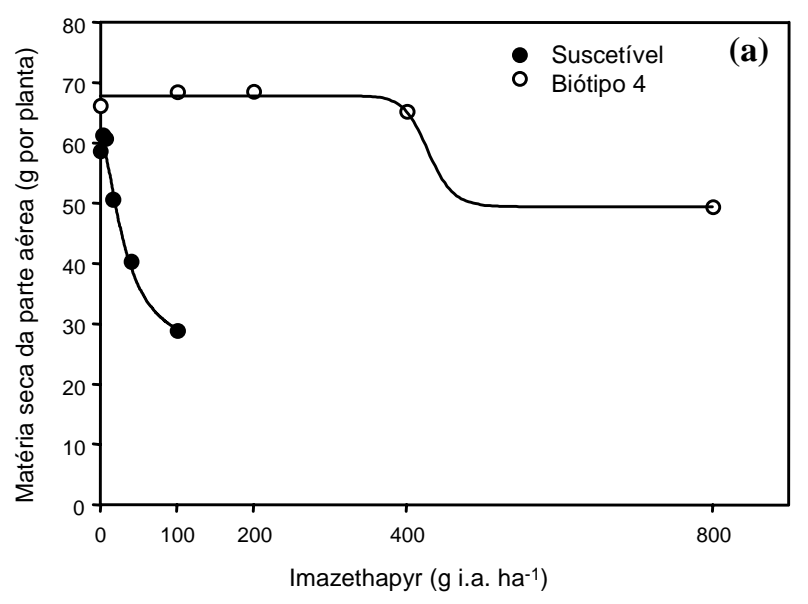

Figura 1 - Resposta dos biótipos S e 4 (R) de E. heterophylla aos herbicidas imazethapyr (a) e fomesafen (b) no ensaio em casa de vegetação.

Planta Daninha, Viçosa-MG, v. 24, n. 3, p. 563-571, 2006

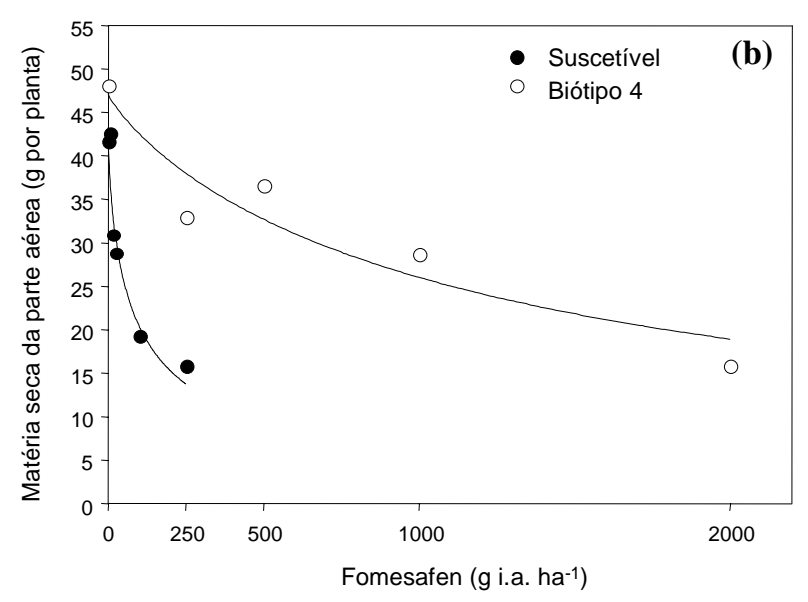

Os dados foram analisados pela análise de variância e, quando o teste de $\mathrm{F}$ foi significativo, fez-se a de regressão polinomial. As médias entre biótipos foram comparadas utilizandose o teste da diferença mínima significativa (DMS) a 5\% de probabilidade.

\section{RESULTADOS E DISCUSSÃO}

Para os dados obtidos no experimento em casa de vegetação, verificou-se ajuste das curvas de dose-resposta da MS da parte aérea do biótipo S e dos biótipos 4 e 23 de EPHHL, para os herbicidas imazethapyr e fomesafen (Figuras 1 e 2). Os parâmetros das equações logísticas do primeiro e do segundo ensaio encontram-se nas Tabelas 1 e 2 .

No primeiro ensaio, em que se compararam as testemunhas sem herbicida dos biótipos $\mathrm{S}$ com 4, a média da MS da parte aérea das plantas $S$ foi de $59 \mathrm{mg}$, enquanto a das plantas do biótipo 4 foi de $66 \mathrm{mg}$ (Figura 1). No segundo ensaio, quando se comparou a testemunha do biótipo S com a do 23, a média da MS da parte aérea das plantas $\mathrm{S}$ foi de $45 \mathrm{mg}$, e a das plantas do biótipo 23, de 46,5 mg (Figura 2). Isso indica que a seleção da resistência múltipla não implicou alterações fisiológicas e bioquímicas nas plantas que resultassem em em relação à população S. No entanto, devem ser desenvolvidos trabalhos mais detalhados para confirmar essa hipótese. redução significativa do seu desenvolvimento 

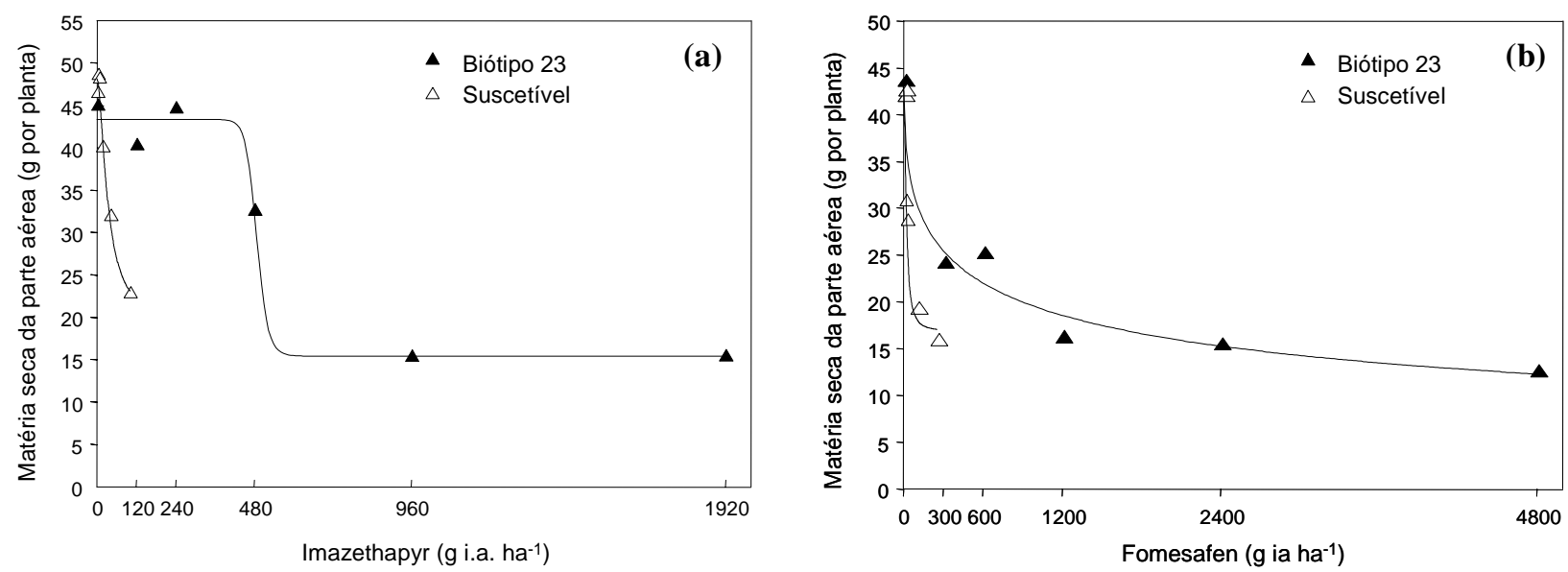

Figura 2 - Resposta dos biótipos S e 23 (R) de E. heterophylla aos herbicidas imazethapyr (a) e fomesafen (b) em ensaio em casa de vegetação.

Tabela 1 - Parâmetros $a, b, c$ e $d$ da equação logística dose-resposta dos biótipos 4 e $\mathrm{S}$, para a variável matéria seca da parte aérea, com os herbicidas testados

\begin{tabular}{|l|c|c|c|c|c|c|c|c|}
\hline \multirow{2}{*}{ Herbicida } & \multicolumn{4}{|c|}{ Biótipo Suscetível } & \multicolumn{4}{c|}{ Biótipo 4 } \\
\cline { 2 - 10 } & $a^{\underline{1}}$ & $b$ & $c$ & $d$ & $a$ & $b$ & $c$ & $d$ \\
\hline Imazethapyr & 36,3 & 1,7 & 32,7 & 24,5 & 18,4 & 26,6 & $>800$ & 49,4 \\
\hline Fomesafen & 43,1 & 0,7 & 21,3 & $-\underline{2}$ & 47,1 & 0,9 & $1.292,0$ & $-\underline{2}-1$ \\
\hline
\end{tabular}

${ }^{1} / a=$ média da resposta da testemunha; $b=$ declividade da curva; $c=$ nível de herbicida que reduz $50 \%$ a matéria seca $\left(\mathrm{I}_{50}\right) ; d=$ média da resposta sob doses elevadas; e ${ }^{1 /}$ equação logística de três parâmetros.

Tabela 2 - Parâmetros $a, b, c$ e $d$ da equação logística dose-resposta dos biótipos 23 e S, para a variável matéria seca da parte aérea, com os herbicidas testados

\begin{tabular}{|l|c|c|c|c|c|c|c|c|}
\hline \multirow{2}{*}{ Herbicida } & \multicolumn{4}{|c|}{ Biótipo Suscetível } & \multicolumn{4}{c|}{ Biótipo 23 } \\
\cline { 2 - 9 } & $a$ & $b$ & $c$ & $d$ & $a$ & $b$ & $c$ & $d$ \\
\hline Imazethapyr & 36,4 & 1,7 & 32,8 & 24,4 & 27,9 & 28,7 & 487,9 & 15,4 \\
Fomesafen & 29,3 & 1,2 & 33,2 & 13,6 & 43,4 & 0,46 & $1.272,0$ & 0,2 \\
\hline
\end{tabular}

${ }^{1} / a=$ média da resposta da testemunha; $b=$ declividade da curva; $c=$ nível de herbicida que reduz $50 \%$ a matéria seca $\left(\mathrm{I}_{50}\right) ; d=$ média da resposta sob doses elevadas.

Com o uso de 100 g i.a. ha-1 de imazethapyr e $250 \mathrm{~g} \mathrm{ha}^{-1} \mathrm{de}$ fomesafen (doses recomendadas pelos fabricantes), houve reduções de 57 e 51\%, respectivamente, da MS da parte aérea do biótipo S, em relação ao biótipo 4 (Figura 1a, b). A utilização de 120 e $300 \mathrm{~g} \mathrm{ha}^{-1}$ de imazethapyr e fomesafen, respectivamente, provocou redução de 43 e $25 \%$ da MS do biótipo S, em relação ao biótipo 23 (Figura 2a, b).
Para o biótipo 4, o fator de resistência (FR) para imazethapyr foi superior a 24 , enquanto para o biótipo 23 ele foi de 15 (Trezzi et al., 2005). Os FRs para fomesafen foram de 62 e 39, para os respectivos biótipos (Trezzi et al., 2005). Todos os valores de FR foram superiores a 1, indicando resistência dos biótipos 4 e 23 aos herbicidas imazethapyr e fomesafen. 
Esses resultados confirmam a resistência múltipla aos herbicidas imazethapyr e fomesafen nos dois biótipos de EPHHL identificados no sudoeste do Paraná. O valor de FR do biótipo 23 foi inferior ao obtido para a MS da parte aérea de EPHHL resistente a imazethapyr, identificado em Assis (SP) (Gelmini et al., 2001), e para percentual de controle de EPHHL resistente a imazethapyr, de Cafelândia (PR) (Gazziero et al., 1998). O FR obtido no biótipo 23 foi similar ao de um biótipo do Mato Grosso do Sul (Gelmini et al., 2005). Os valores de FR para imazethapyr identificados por Gelmini et al. (2001, 2005) foram similares aos obtidos para o biótipo 4 do presente ensaio.

Os valores de FR para fomesafen identificados no presente ensaio foram muito superiores aos valores de 8 e 6,2 observados em biótipos de Amaranthus spp. resistentes a fomesafen, respectivamente, nos estados do Kansas e Illinois, Estados Unidos (Shoup et al., 2003; Padzoldt et al., 2005). Dayan et al. (1996) aventaram a hipótese de que plantas podem ser selecionadas para resistência a inibidores da PROTOX por meio de mecanismos como modificações na absorção, translocação e metabolização dos herbicidas. Até o momento, para os biótipos de Amaranthus spp. essas hipóteses foram descartadas (Li et al., 2004), porém há indícios de que uma mutação na enzima PROTOX seja a responsável pela resistência nessa espécie (Tranel et al., 2006). Não existem estudos, até o momento, que identifiquem as causas da resistência nos biótipos de EPHHL com resistência múltipla identificados na região sudoeste do Paraná.

Os valores de FR elevados para herbicidas inibidores da ALS e da PROTOX, identificados neste trabalho, sugerem que o mecanismo de resistência esteja associado ao local de ação enzimático alterado. De fato, biótipos de EPHHL com resistência a inibidores da ALS identificados no Rio Grande do Sul demonstram que há alteração no local de ação da enzima ALS, caráter dominante e codificado no núcleo (Vargas et al., 2001). No entanto, a identificação de resistência cruzada a outros herbicidas inibidores da PROTOX, pertencentes às ftalamidas, triazolinonas e aos difeniléteres, mostrou percentuais elevados de controle de plantas dos biótipos 4 e 23 de
EPHHL pela utilização de sulfentrazone e flumioxazin, os únicos aplicados em pré-emergência das plantas (Trezzi et al., 2005). Isso indica que a forma de absorção dos inibidores da PROTOX pode estar relacionada ao mecanismo de resistência nesses biótipos.

Quanto aos resultados observados nos ensaios em laboratório, para corrigir diferenças intrínsecas entre os biótipos, os valores das variáveis dependentes obtidos nessa etapa foram transformados em valores percentuais em relação às testemunhas sem herbicida (Figuras 3 e 4).

Em ambos os ensaios, com imazethapyr e fomesafen, a análise da variância do percentual de germinação final não identificou interação entre biótipo e concentrações de herbicidas, tampouco diferenças entre os biótipos S e 4 . Houve redução abrupta e até próximo da ausência de capacidade germinativa das sementes de ambos os biótipos de EPHHL a partir das concentrações de $80 \mathrm{mg} \mathrm{L}^{-1}$ de imazethapyr, enquanto para fomesafen esse efeito somente foi observado com a utilização de $5.000 \mathrm{mg} \mathrm{L}^{-1}$ (dados não mostrados). Bioensaio germinativo conduzido por Carvalho et al. (2004) demonstrou que o herbicida metribuzin inibiu completamente a germinação de Bidens spp. em concentrações superiores a $300 \mathrm{mg} \mathrm{L}^{-1}$. No mesmo trabalho, no entanto, níveis até $10.000 \mathrm{mg} \mathrm{L}^{-1}$ de imazaquin, inibidor da ALS como imazethapyr, não inibiram a germinação das sementes de Bidens spp. (Carvalho et al., 2004) - diferenças que podem ser atribuídas a vários fatores, como toxicidade do princípio ativo e sensibilidade diferencial das espécies-alvo, entre outros.

No ensaio com imazethapyr, a análise da variância indicou significância da interação entre biótipo e níveis de herbicida, para o comprimento da radícula (COMRA), e efeitos simples de biótipo e níveis de herbicidas, para o comprimento da parte aérea (COMPA). No ensaio com fomesafen, a análise da variância indicou significância da interação entre biótipo e níveis do herbicida para COMRA e COMPA.

A relação entre os niveis de herbicidas e o COMRA/COMPA não foi adequadamente ajustada através da equação logística. Assim, utilizou-se a regressão polinomial quadrática para efetuar o ajuste entre variável dependente 
e independente. Em geral, COMRA foi um parâmetro mais sensivel para avaliação do crescimento de EPHHL em resposta a imazethapyr do que COMPA (Figura 3a, b). No entanto, ambos os comprimentos foram completamente inibidos com a utilização da dose máxima de imazethapyr (320 $\left.\mathrm{mg} \mathrm{L}^{-1}\right)$. Em média, o COMPA (\% da testemunha sem imazethapyr) do biótipo 4 foi de $64 \%$, valor superior ao do biótipo S, de $52 \%$ (Figura 3a). As diferenças entre COMRA (\% da testemunha) dos biótipos 4 e S variaram em função da concentração de imazethapyr empregada (Figura 3b). Apenas nas concentrações de 40 e $160 \mathrm{mg} \mathrm{L}^{-1}$ foi identificada, através de significância estatística, a superioridade do crescimento radicular do biótipo 4 em comparação ao S.

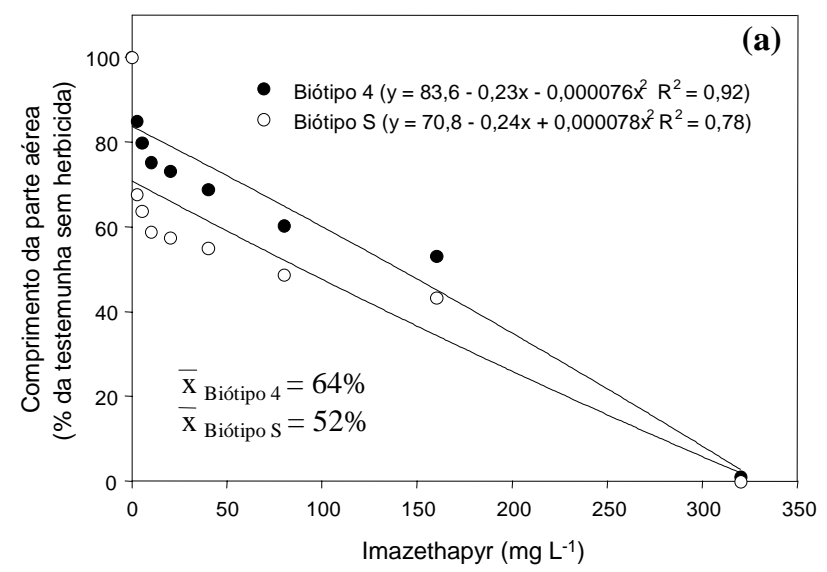

Tanto COMPA quanto COMRA revelaramse parâmetros sensiveis para avaliação das diferenças de crescimento dos biótipos 4 e S de EPHHL em resposta a fomesafen (Figura 4a, b). Assim como ocorreu com imazethapyr, essas variáveis foram completamente inibidas com a utilização da dose máxima de fomesafen (5.000 $\mathrm{mg} \mathrm{L}^{-1}$ ). No entanto, as diferenças entre COMPA e COMRA (\% da testemunha) variaram em função do nível de fomesafen utilizado (Figura 4a, b). Em todas as concentrações, exceto as de 1.250 e $5.000 \mathrm{mg} \mathrm{L}^{-1}$ de fomesafen, o COMRA do biótipo 4 foi superior ao do biótipo S (Figura 4a). O COMPA do biótipo 4 de EPHHL foi superior ao do biótipo $\mathrm{S}$ nas concentrações de $625,1.250,2.500$ e $3.125 \mathrm{mg} \mathrm{L}^{-1}$ (Figura 4b). O fato de que as diferenças de resposta

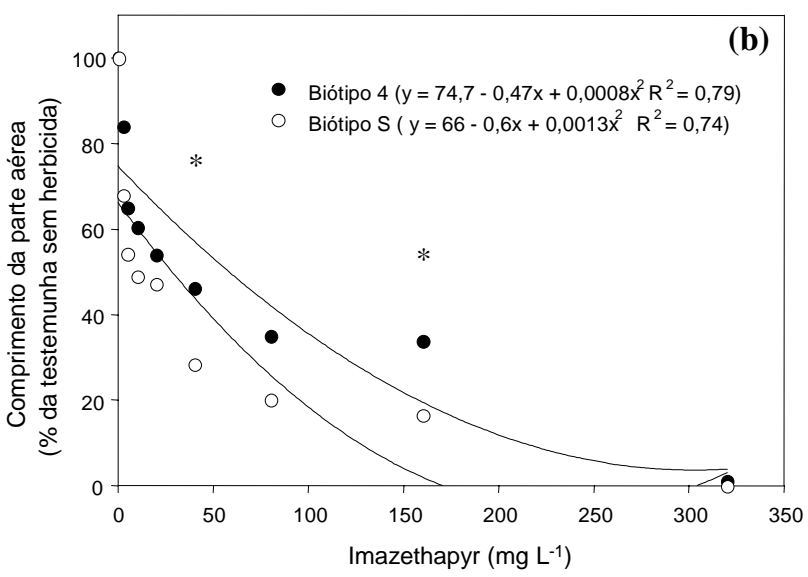

Figura 3 - Efeito do herbicida imazethapyr sobre o comprimento da parte aérea (a) e da radícula (b) dos biótipos 4 (R) e S de E. heterophylla em bioensaio em laboratório. Asteriscos indicam diferença significativa pelo teste DMS a 5\% de probabilidade entre biótipos, no nível do herbicida.
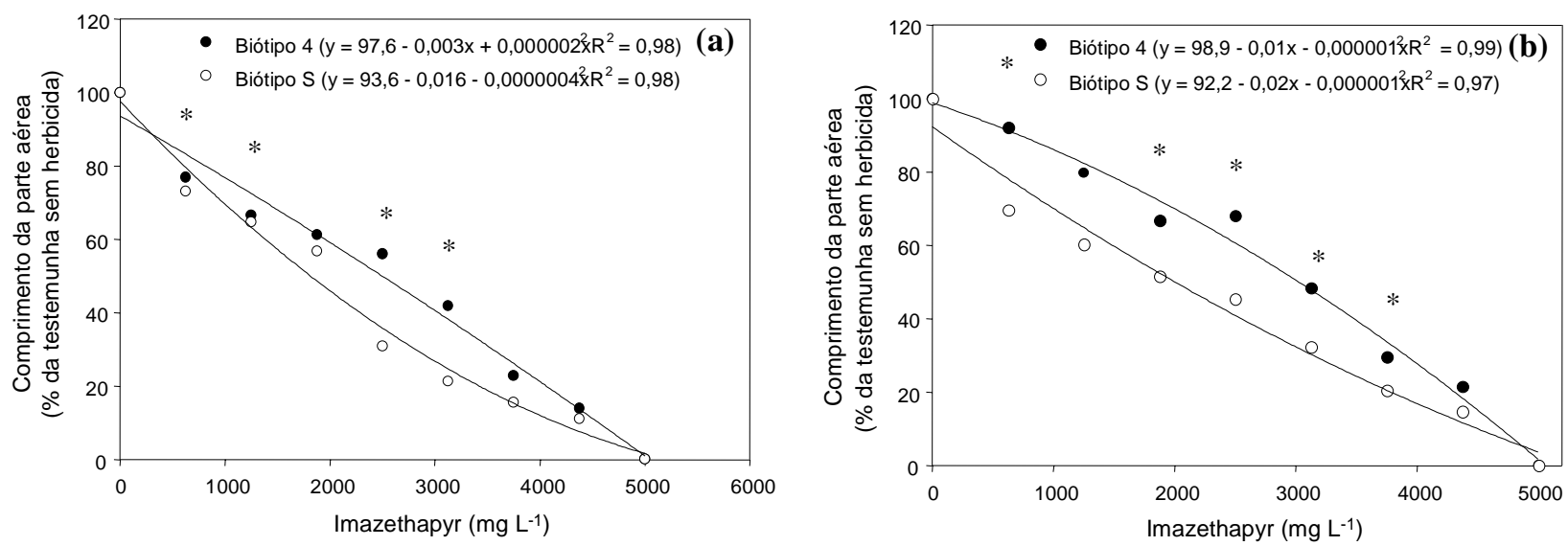

Figura 4 - Efeito do herbicida fomesafen sobre o comprimento da parte aérea (a) e da radícula (b) dos biótipos 4 (R) e $\mathrm{S}$ de E. heterophylla em bioensaio em laboratório. Asteriscos indicam diferença significativa pelo teste DMS a 5\% de probabilidade entre biótipos, no nível do herbicida. 
entre os biótipos $\mathrm{R}$ e $\mathrm{S}$ foram significativas apenas em algumas concentrações de herbicidas pode ser explicado pelo pequeno número de graus de liberdade do ensaio, em razão do número reduzido de repetições.

Sucesso na diferenciação de biótipos de Phalaris minor, Lolium rigidum e Alopercus myosuroides resistentes e suscetiveis a inibidores da ACCase através do uso de bioensaios com sementes também foi obtido por Tal et al. (2000). Por meio dessa técnica, Carvalho et al. (2004) identificaram diferenças entre três biótipos de Bidens spp. resistentes e um suscetivel a inibidores da ALS. Nesse trabalho, eles apontaram a possibilidade de diferenciar biótipos $\mathrm{R}$ de $\mathrm{S}$ através do ajuste de curvas de dose-resposta, utilizando a variável porcentagem de plântulas completamente anormais.

O crescimento diferencial das partes aérea e radicular entre os biótipos $\mathrm{R}$ e $\mathrm{S}$ em resposta a imazethapyr e fomesafen é mais um indicativo de que há resistência a esses herbicidas no biótipo 4, permitindo especular que o mecanismo envolvido não esteja relacionado à absorção ou translocação do herbicida inibidor da PROTOX, pois nas condições em que foram conduzidos os ensaios esses processos são maximizados.

Comprovou-se, neste trabalho, com a utilização de curvas de dose-resposta em ensaios em casa de vegetação e laboratório, a existência de biótipos de EPHHL com resistência múltipla aos herbicidas inibidores da ALS e da PROTOX na região sudoeste do Paraná. Esses são os primeiros casos de resistência múltipla no Brasil e os primeiros de resistência múltipla de biótipos de EPHHL no mundo. Os crescimentos das partes aérea e radicular revelaramse parâmetros mais adequados do que o percentual final de germinação para discriminar diferenças entre biótipos com resistência múltipla de biótipos S. No entanto, somente algumas doses dos herbicidas permitiram essa diferenciação, podendo ser empregadas para tal finalidade.

\section{AGRADECIMENTOS}

Aos acadêmicos do Curso de Agronomia da UTFPR Cleverson Felippi, Márcio Coppini e Odílio Maeda, pelo apoio na realização das determinações em casa de vegetação e laboratório.

\section{LITERATURA CITADA}

BECKIE, H. J. et al. Screening for herbicide resistance in weeds. Weed Technol., v. 4, n. 2, p. 428-445, 2000.

CARVALHO, S. J. P. et al. Identificação de biótipos de Bidens spp. resistentes aos inibidores da ALS através de teste germinativo. Planta Daninha, v. 22, n. 3, p. 411-417, 2004.

CHRISTOFFOLETI, P. J. et al. Imidazolinone resistant Bidens pilosa biotypes in the brazilian soybean areas. In: MEETING OF THE WSSA, 1996, Norfolk. Abstracts... Champaign: WSSA, 1996. p. 10.

DAYAN, F. E.; WEETE, J. D.; HANCOCK, H. G. Physiological basis for differential sensitivity to sulfentrazone by sicklepod (Senna obtusifolia) and coffee senna (Cassia occidentalis). Weed Sci., v. 44, n. 1, p. 12-17, 1996.

GAZZIERO, D. L. P. et al. Resistência de amendoim-bravo aos herbicidas inibidores da ALS. Planta Daninha, v. 16, n. 2, p. 117-125, 1998.

GELMINI, G. A. et al. Resistência de biótipos de Euphorbia heterophylla L. aos herbicidas inibidores da enzima ALS utilizados na cultura da soja. Bragantia, v. 60, n. 2, p. 93-99, 2001.

GELMINI, G. A. et al. Resistance of Euphorbia heterophylla L. to ALS-inhibiting herbicides in soybean. Sci. Agric., v. 62, n. 5 , p. $452-457,2005$.

HEAP, I. International survey of herbicide-resistant weeds. Disponível em: <www.weedscience.org>. Acesso em: 15 jan. 2006.

LI, J. et al. Physiological basis for resistance to diphenyl ether herbicides in common waterhemp (Amaranthus rudis). Weed Sci., v. 52, n. 3, p. 333-338, 2004.

MEROTTO Jr., A.; VIDAL, R. A. Herbicidas inibidores da PROTOX. In: VIDAL, R. A.; MEROTTO Jr., A. (Eds.). Herbicidologia. Porto Alegre: Evangraf, 2001. p. 69-86.

MONQUEIRO, P.; CHRISTOFFOLETI, P. J.; DIAS, C. T. S. Resistência de plantas daninhas aos herbicidas inibidores da ALS na cultura da soja (Glycine max). Planta Daninha, v. 18, n. 3, p. 419-425, 2000.

NOLDIN, J. A.; EBERHARDT, D. S.; KNOBLAUCH, R. Resistência aos herbicidas em Sagittaria montevidensis: primeiras evidências. In: CONGRESSO BRASILEIRO DE ARROZ IRRIGADO, 1., 1999, Pelotas. Anais... Pelotas: Embrapa, 1999. p. 566-567.

NOLDIN, J. A.; EBERHARDT, D. S.; RAMPELOTTI, F. T. Cyperus difformis L. resistente a herbicidas inibidores da ALS em Santa Catarina. In: CONGRESSO BRASILEIRO DA CIÊNCIA DAS PLANTAS DANINHAS, 23., 2002.

Gramado. Anais... Londrina: Sociedade Brasileira da Ciência das Plantas Daninhas, 2002a. p. 198. 
NOLDIN, J. A.; EBERHARDT, D. S.; RAMPELOTTI, F. T. Fimbristylis miliacea (L.) Vahl resistente a herbicidas inibidores da ALS em Santa Catarina. In: CONGRESSO BRASILEIRO DA CIÊNCIA DAS PLANTAS DANINHAS, 23., 2002, Gramado. Anais... Londrina: Sociedade Brasileira da Ciência das Plantas Daninhas, 2002b. p. 199.

PATZOLDT, W. L.; TRANEL, P. J.; HAGER, A. G. A waterhemp (Amaranthus tuberculatus) biotype with multiple resistance across three herbicide sites of action. Weed Sci., v. 53, n. 1, p. 31-36, 2005.

SEEFELDT, S. S.; JENSEN, J. E.; FUERST, E. P. Loglogistic analysis of herbicide dose-response relationships. Weed Technol., v. 9, n. 2, p. 218-227, 1995.

SHOUP, D.E.; AL-KHATIB, K.; PETERSON, D. A. Common waterhemp (Amaranthus rudis) resistance to protoporphyrionogen oxidase-inhibiting herbicides. Weed Sci., v. 51, n. 2, p. 145-150, 2003.

TAL, A.; KOTOULA-SYKA, E; RUBIN, B. Seed-bioassay to detect grass weeds resistant to acetyl coenzyme A carboxylase inhibiting herbicides. Crop Protec., v. 19, p. 467-472, 2000.
TRANEL, P. J.; PADZOLDT, W. L.; HAGER, A. G. An altered target site confers resistance to PPO-inhibiting herbicides in a waterhemp biotype. In: ANNUAL MEETING OF THE WEED SCIENCE SOCIETY OF AMERICA, 46., 2006, New York. Abstracts... Champaign: WSSA, 2006. p. 256.

TREZZI, M. M. et al. Multiple resistance of acetolactate synthase and protoporphyrinogen oxidase inhibitors in Euphorbia heterophylla biotypes. J. Environ. Sci. HealthPart B, v. 40, p. 101-109, 2005.

VARGAS, L.; BORÉM, A.; SILVA, A.A. Herança da resistência aos herbicidas inibidores da ALS em biótipos da planta daninha Euphorbia heterophylla. Planta Daninha, v. 19, p. 331-336, 2001.

VIDAL, R. A.; MEROTTO Jr., A. Resistência de amendoim bravo (Euphorbia heterophylla L.) aos herbicidas inibidores da enzima acetolactato sintase. Planta Daninha, v. 17, n. 3, p. 367-373, 1999.

VOLL, E. et al. Relações entre germinação de sementes de espécies de plantas daninhas e uso da condutividade elétrica. Planta Daninha, v. 21, n. 1, p. 181-189, 2003. 